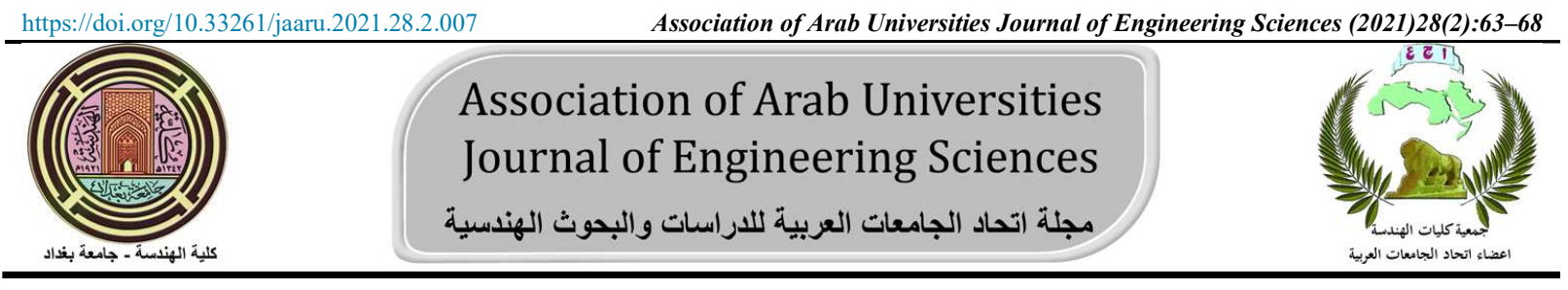

تأثثير معالجة الإرجاع وإعادة التعتيق على صلادة ومقاومة الثد و البنية المجهرية لسبيكة الألمنيوم 7075

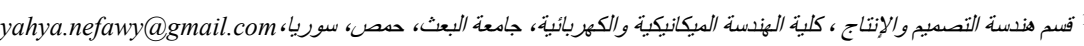

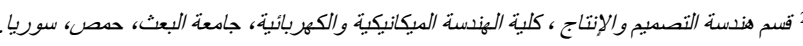

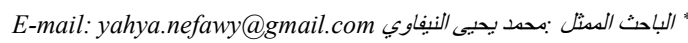
نشر في: 30 حزيران 2021

الخلاصة ـ تحسن معالجة الإرجاع و إعادة التعتيق الحرارية (RRA) مقاومة الثد لسبائك الألمنيوم. في هذا البحث لارب استة

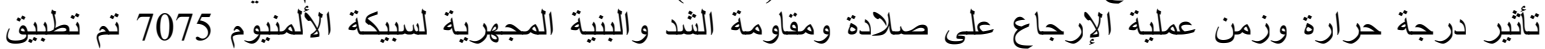

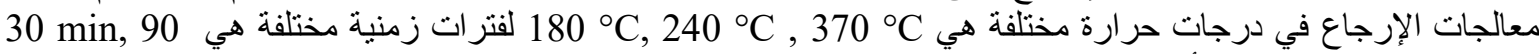

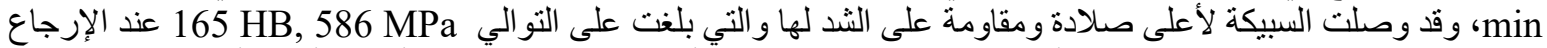

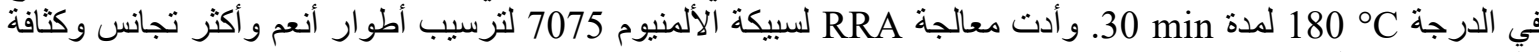

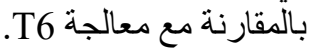

الكلمات الرئيسية _ " سبيكة الألمنيوم عالية المقاومة 7075" ، " الإرجاع و إعادة التعتيق" ، " صلادة " ، " مقاومة الثد " ، " بنية مجهرية " ، " حيود الأشعة السينية "ليكة الالمنيوم

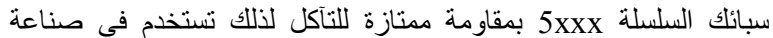

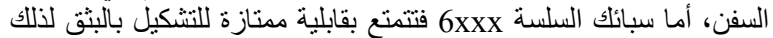

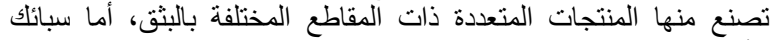

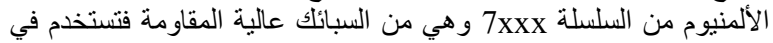

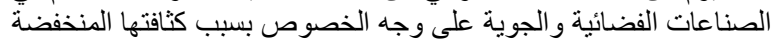

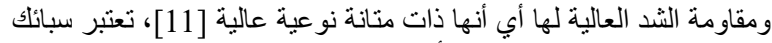

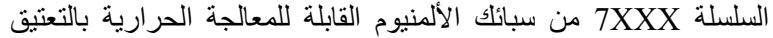
الاصطناعي وتأتي المقاومة العالية لسبيكة الألمنيوم 7075 بفعل مناطقة اللفئة و'(MgZn ورواسب الطور شبه المستقر

الغني بالمغنيزيوم و الزنك [5]

$$
\text { 2. الدراسات السابقة }
$$

تتغير خواص سبيكة الألمنيوم 7075 وبنيتها المجهرية والأطوار المنشكلة

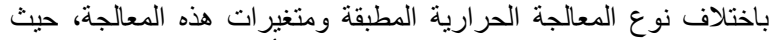

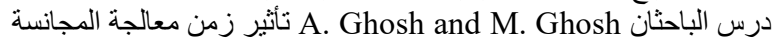
على البنية المجهرية والاطوار المتشكلة وخواص سبيكة الألمنيوم 7075

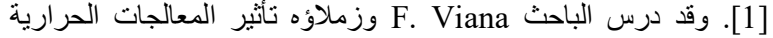

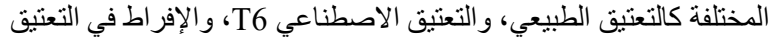

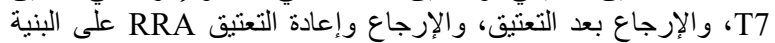

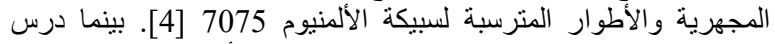
الباحثان Mavropoulos and Skolianos تأثثير هذه الماند المعالجات

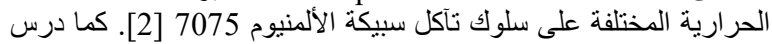

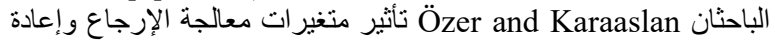

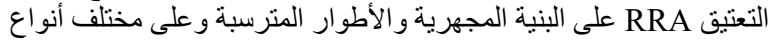
التآكل لسبيكة الألمنيوم 7075 [6]

إن مقاومة سبيكة الألمنيوم 7075 على الثند تبلغ قيمتها العظمى عند تطبيق

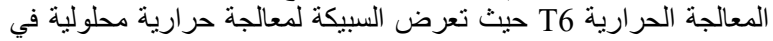

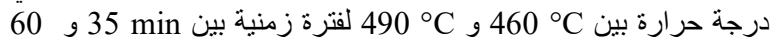

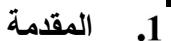

ينتشر فلز الألمنيوم في صخور الأرض بنسبة تفوق كل الفلزات الأخرى،

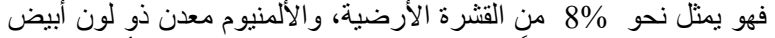

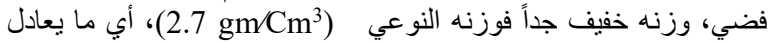

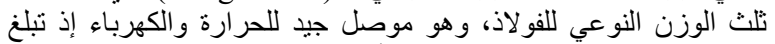

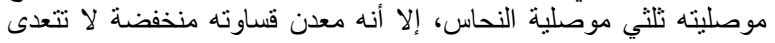

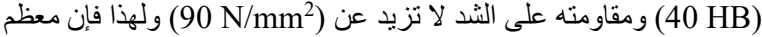

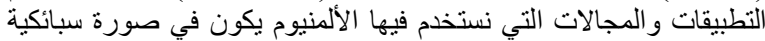

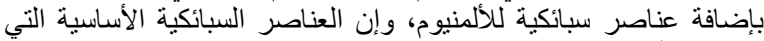

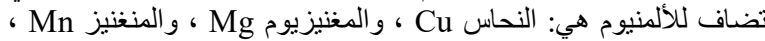

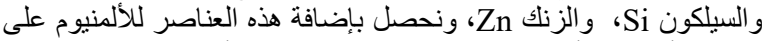

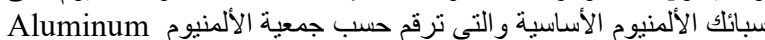
Association

$$
\begin{aligned}
& \text { 1xxx } \\
& \text { 2xxx } \\
& \text { 3xxx } \\
& \text { 4xxx } \\
& \text { 5xxx } \\
& \text { 6xxx } \\
& \text { 7xxx } \\
& \text { 8xxx }
\end{aligned}
$$

تتميز كل سلسلة من هذه السلاسل بخاصية تميزها عن باقي السلاسل

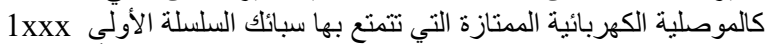

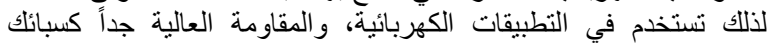

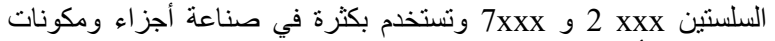

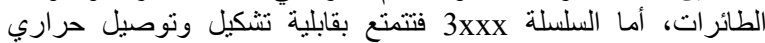
فتستخدم في تصنيع علب المشروبات الغازية والمبادلاتلات الحر ارية، وتنتئع 


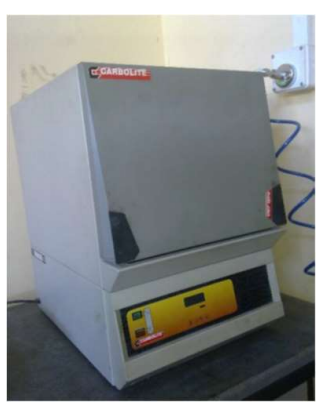

الثكل 1: الفرن الكهربائي المستخدم في المعالجات الحررية

\section{النتائج والمناقشة}

\section{.4}

تم إجراء اختبار ات على العينات المعالجة حر ارياً بمختلف الحالات لدراسة الإنة تأثير درجات حرارة وأزمنة معالجة الإرجاع على خواص سبيكة الألمنيوم

$$
\text { دراسة خواص الثد }
$$

تم إجراء اختبار الثند في مختبر خواص المواد في كلية الهندسة الميكانيكية والكهربائية بجامعة البعث وفق المواصفة

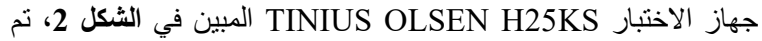

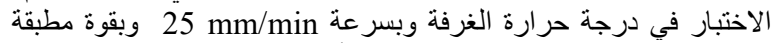

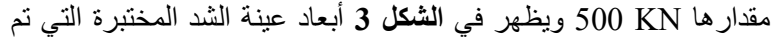
الحصول عليها من السبيكة بعد عمليات التشغيل الميكانيكي المناسبة (خراطة)، وقد تم تطبيق المعالجات الحرارية على العينات بعد التيكاتيكي التهاء عمليات التشغيل.

يظهر في الشكل 4 تغيرات مقاومة الثد لسبيكة الألمنيوم 7075 بتغير

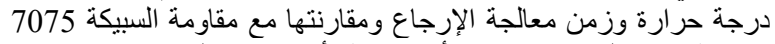

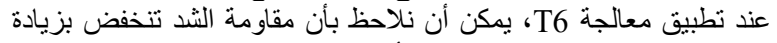

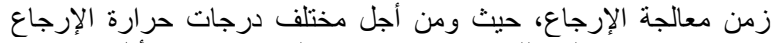

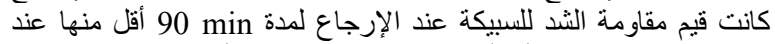

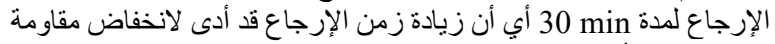

الثد لسبيكة الألمنيوم 7075.

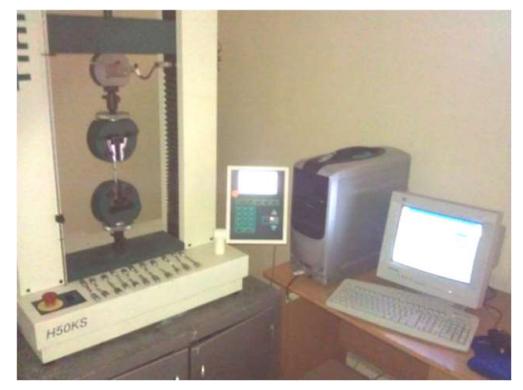

الثكل 2: جهاز اختبار الثد المستخدم

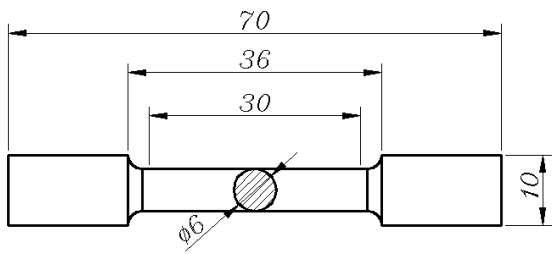

الثكل 3: أبعاد عينة الثد بـ mm min

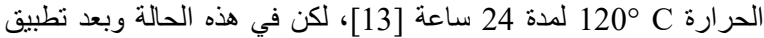

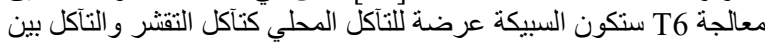

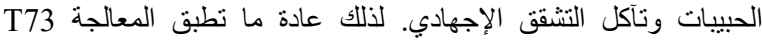

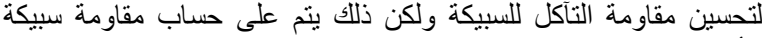

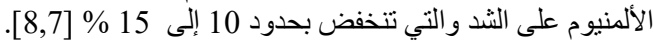

في حين أن معالجة الإرجاع و إعادة التعنيق RRA التي طور ها Cina في

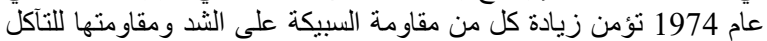

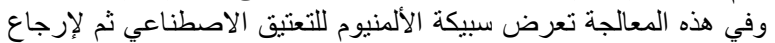

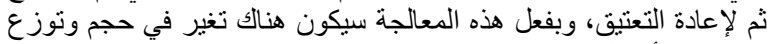

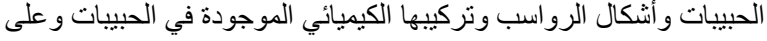

حدودها [3].

وسيتم في هذا البحث دراسة تأثير تغير درجة حرارة وزمن عملية الإرجاع

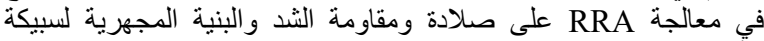
الألمنيوم عالية المقاومة 7075.

\section{3. منهجية البحث}

كانت سبيكة الألمنيوم 7075 المستخدمة في البحث على شكل لوح بسماكة

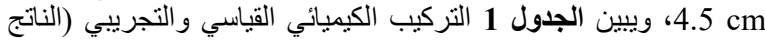
بعد تحليل السبيكة 7075 بجهاز التحليل الطيفي السبيكترومتر ).

\begin{tabular}{|c|c|c|}
\hline التركيب التجريبي & التركيب القياسي & العنصر \\
\hline 0.085 & $<0.4$ & السيليكون Si \\
\hline 0.05 & $<0.2$ & التيتانيو مi Ti \\
\hline 0.18 & $0.18-0.28$ & الكروم Cr \\
\hline 0.17 & $<0.5$ & Fe الحديد \\
\hline 1.8 & $1.2-2$ & النحاس Cu \\
\hline 2.35 & $2.1-2.9$ & المغنزيوم Mg \\
\hline 0.01 & $<0.3$ & المنغنيز Mn \\
\hline 5.8 & $5.1-6.1$ & الزنك Zn \\
\hline Bal. & Bal. & الألمنيوم A1 \\
\hline
\end{tabular}

أستخدم الفرن الكهربائي المبين في الثكل 1 لنطبيق المعالجات الحرارية

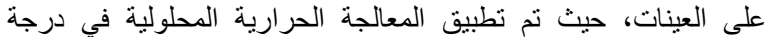

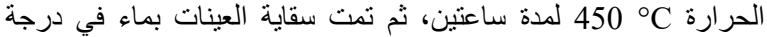

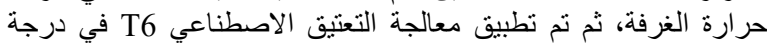

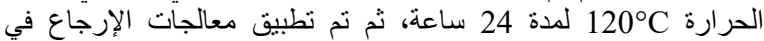
درجات حرارة مختلفة هي $180^{\circ} \mathrm{C}, 240^{\circ} \mathrm{C}, 370^{\circ} \mathrm{C}$ لفترات الات زمنية

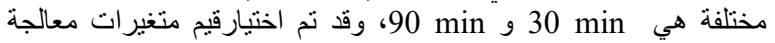

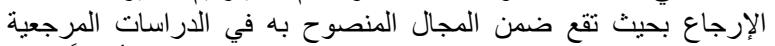

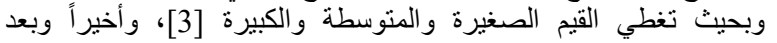

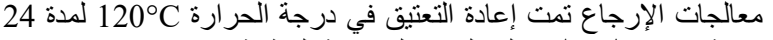
ساعة، ويبين الجدول 2 المعالجات الحراد ارية المطبقة ومتغير اتها.

الجدول 2: متغيرات المعالجات الحرارية المطبقة

\begin{tabular}{|c|c|c|}
\hline 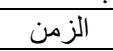 & درجة الحرارة & \\
\hline 2 hour & $450{ }^{\circ} \mathrm{C}$ & 1- معالجة حر ارية محلولية \\
\hline 24 hour & $120^{\circ} \mathrm{C}$ & 2- تعتيق اصطناعي T6 \\
\hline $\begin{array}{l}30 \mathrm{~min} \\
90 \mathrm{~min}\end{array}$ & $\begin{array}{l}180^{\circ} \mathrm{C} \\
240^{\circ} \mathrm{C} \\
370^{\circ} \mathrm{C}\end{array}$ & 3- إرجاع \\
\hline 24 hour & $120^{\circ} \mathrm{C}$ & 4- إعادة التعتيق الاصطناعج \\
\hline
\end{tabular}




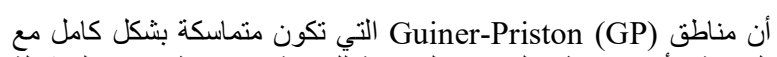

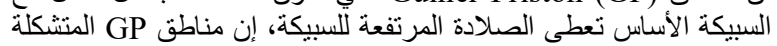

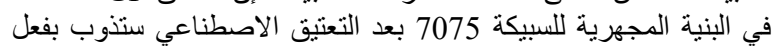

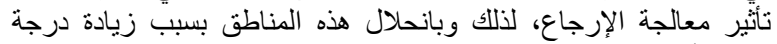
حر ارة أو مدة معالجة الإرجاع ستنخفض قيم الصلادة ومقاومة الثد.

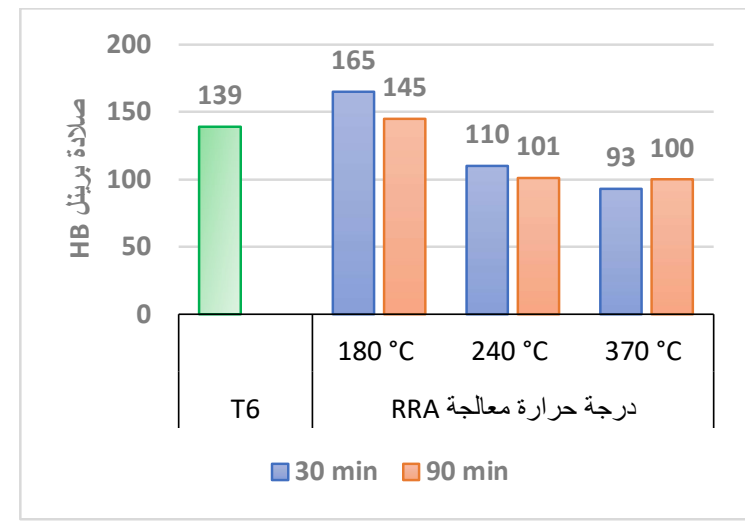

$$
\text { الثكل 6: تغير صلادة برينل للسبيكة الإرجاعة } 7075 \text { بتغير درجة حرارة وزمن }
$$$$
\text { لدراسة البنية المجهرية }
$$

4.3

إن تأثير معالجة الإرجاع وإعادة التعتيق على مقاومة الثد للسبيكة 7075

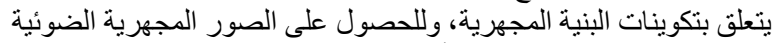

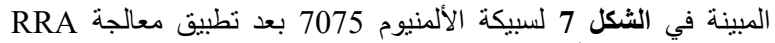
بدرجات حرارة و أزمنة مختلفة وفق المواصفة ASTM-E3

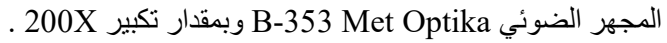

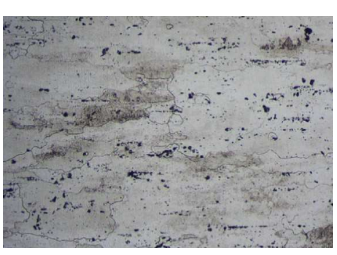

$180^{\circ} \mathrm{C} 90 \mathrm{~min}$

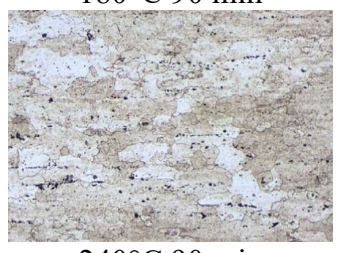

$240^{\circ} \mathrm{C} 90 \mathrm{~min}$

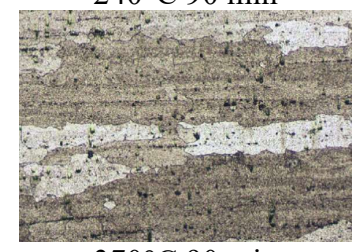

$370^{\circ} \mathrm{C} 90 \mathrm{~min}$

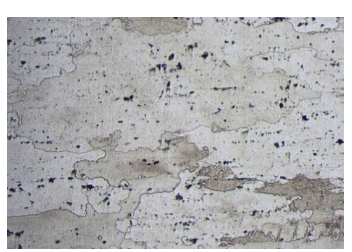

$180^{\circ} \mathrm{C} 30 \mathrm{~min}$

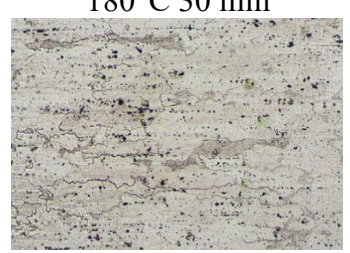

$240^{\circ} \mathrm{C} 30 \mathrm{~min}$

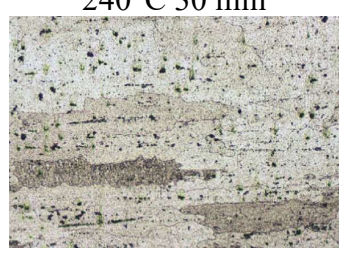

$370^{\circ} \mathrm{C} 30 \mathrm{~min}$

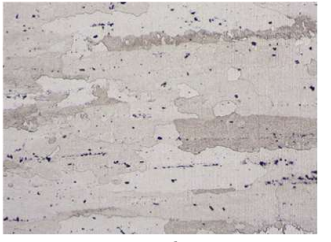

T6

الثكل 7: الصور المجهر الضوئي لعينات من سبيكة الألمنيوم 7075
كما يمكن أن نلاحظ أيضاً بأن زيادة درجة حرارة معالجة الإرجاع قد أدى زلإن

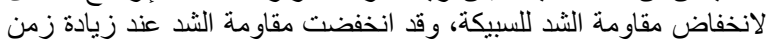

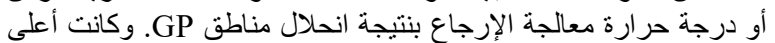

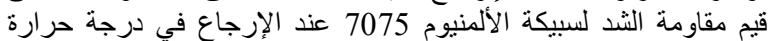

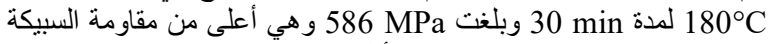

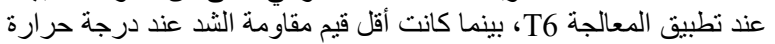

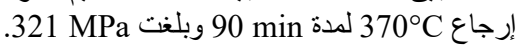

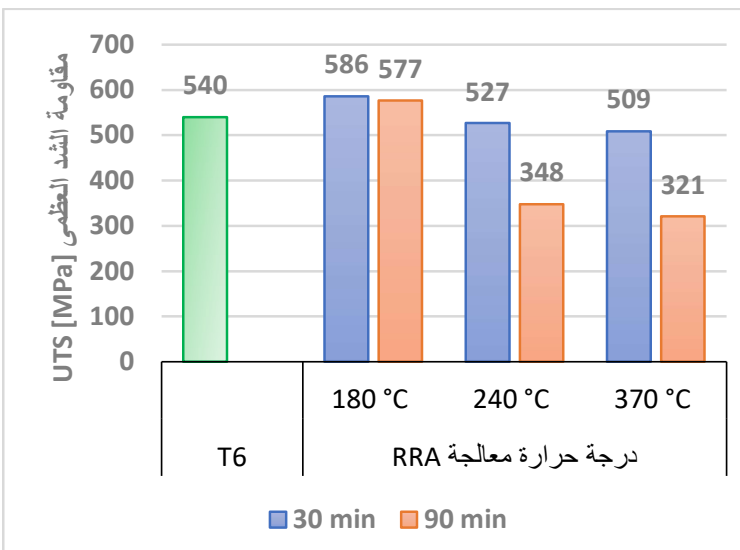

الثكل 4: تغير مقاومة الثد للسبيكة 7075 بتغير درجة حرارة وزمن معالجة الإرجاع - معاع

دراسة الصلادة

تم إجراء اختبار الصلادة وفق المواصفة ASTM-E10 بواسطة جهاز قياس صلادة برينل من نوع ERNSL طرار المراز

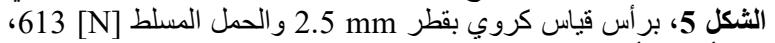
وقد أخذت أكثر من قراءة كربة لكل عينة من مناطق مختلفة لتحديد القيمة المتوسطة لقساوتها.

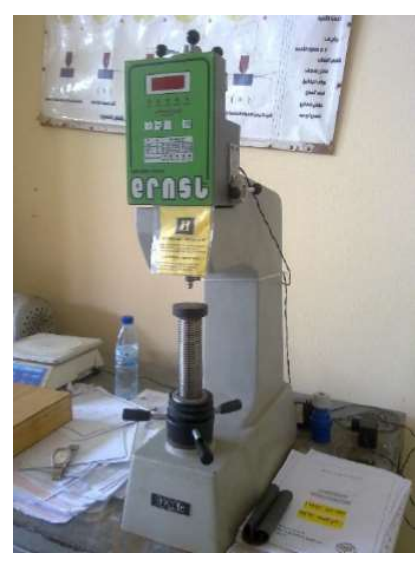

الشكل 5: جهاز قياس صلادة برينل

يظهر في الشكل 6 تغيرات صلادة برينل لسبيكة الألمنيوم 7075 بتغير

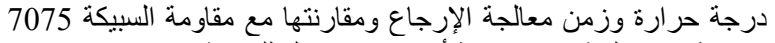

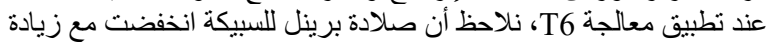

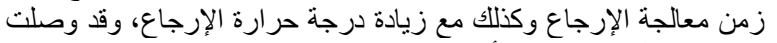

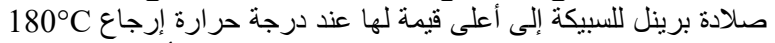

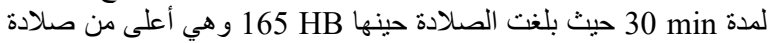
السبيكة عند تطبيق معالجة T6 بلغت 
[6] RRA بعد معالجة T6 (b) بعد الألمنيوم، (a) بعالجة ونلاحظ من الثكل 8 وجود اختلاف في بنية سبيكة الألمنيوم 7075 والجية الجية

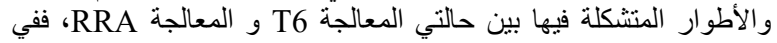

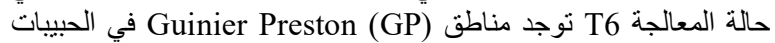

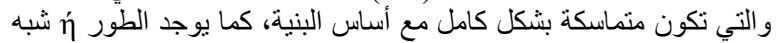
المنمأسك، ومن ناحية أخرى فإن الطور على شكل شبكة أطوار مستمرة موجودة على حدود الطبيبات، الثكل -8)

نتيجة معالجة الإرجاع و وإعادة التعتيق سيكون هناك تغيرات في البنية

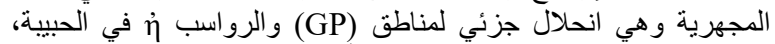

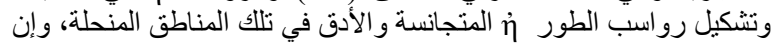

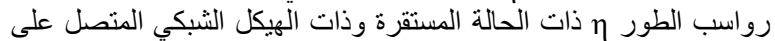

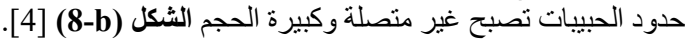

وبالتالي في نهاية عملية الإرجاع و إعادة التعتيق RRA تتشكل في الحبيبات

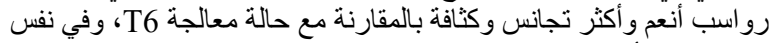

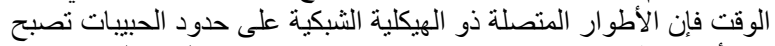

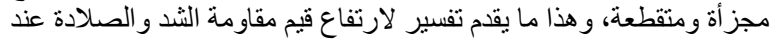
تطبيق معالجة الإرجاع و إعادة التعتيق (RRA) عما كانت عليه في معالجة

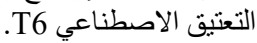

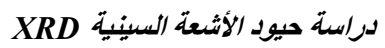

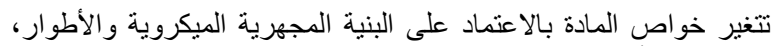

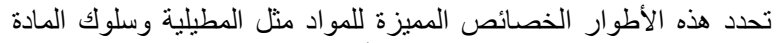

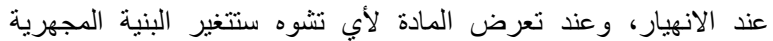

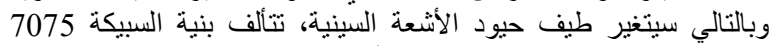
المعرضة للتعتيق الاصطناعي بشكل أساني من الطينة الطورين Al و والطور

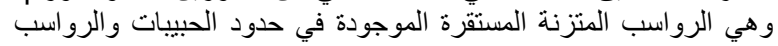
شبه المتماسكة في الحبيية للسبيكة 7075 وهي ذات ذات بنية كيميائية هي

$$
\text { .[10,9] } \mathrm{MgZn}_{2}
$$

تم إجراء اختبارات حيود الأشعة السينية على العينات المعالجة بالمعالجتين

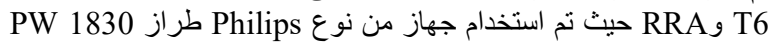
المبين في الشكل و6 و 9

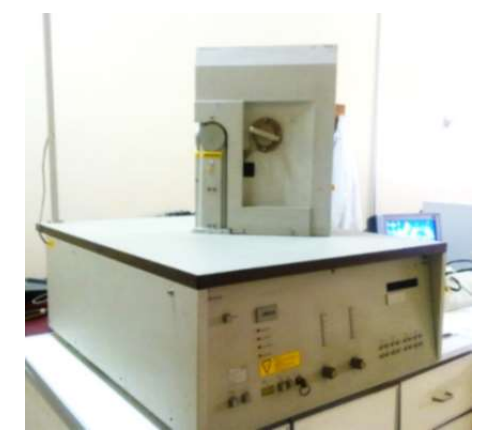

الثكل 9: جهاز اختبار حيود الأشعة السينية XRD

أظهرت نتائج تحليل طيف حيود الأشعة السينبة XRD المبينة في الثكل

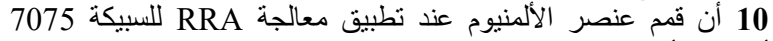
أصبحت أقصر عما كانت عليه عند معالجة T6، في حين ازداد تركيز قمم الطور MgZn الإرجاع و إعادة التعتيق RRA قد عملت على تكوين روالت رواسب أكثر كثافة.

$$
\text { 200X بأزمنة ودرجات حرارة مختلفة عند تكبير RRA بعد معالجة }
$$

تمت عملية الثحذ باستخدام جهاز الثحذ نصف الآلي وأوراق الثحذ المائي

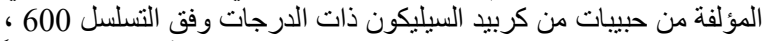

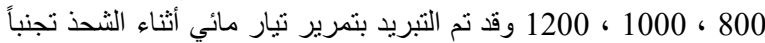
لأي تغير ات في البنية بسبب الحر ارة الناتجة.

وللحصول على سطح مصقول بدون خدوش للعينة تم استخدام معجون

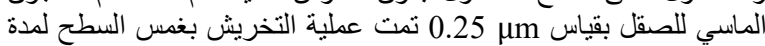
8 ثوان في محلول الإظهار Keller's Reagent وهو ذو لتو التركيب التالي لئي

$1 \mathrm{Cm}^{3} \mathrm{HF}, 1.5 \mathrm{Cm}^{3} \mathrm{HCL}, 2.5 \mathrm{Cm}^{3} \mathrm{HNO}_{3}, 95 \mathrm{Cm}^{3} \mathrm{H}_{2} \mathrm{O}$

تعطي صور المجهر الضوئي فكرة عامة عن التغيرات الحاصلة في البنية

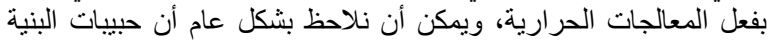

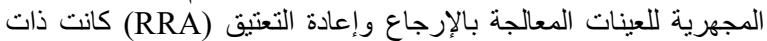

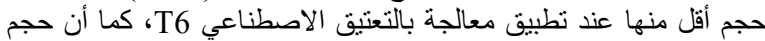

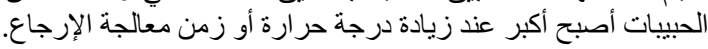

وللحصول على معلومات أكثر لابد من تحليل البنية باستخدام المجهر

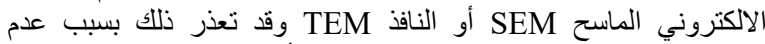
جاهزية هذه المجاهر لدينا في الوقت الذاني الذاني أعدت فيه الدراسة، ويمكن

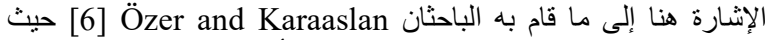

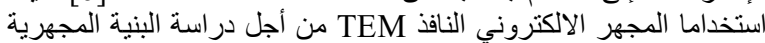

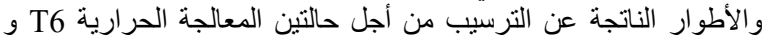

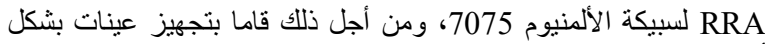

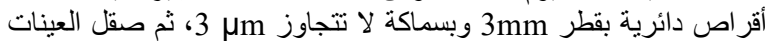

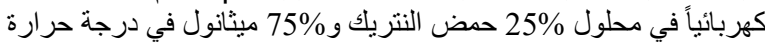

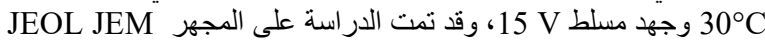

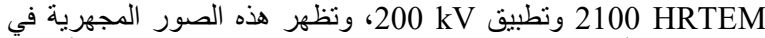

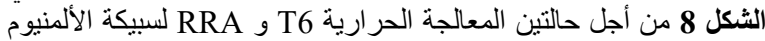

.7075

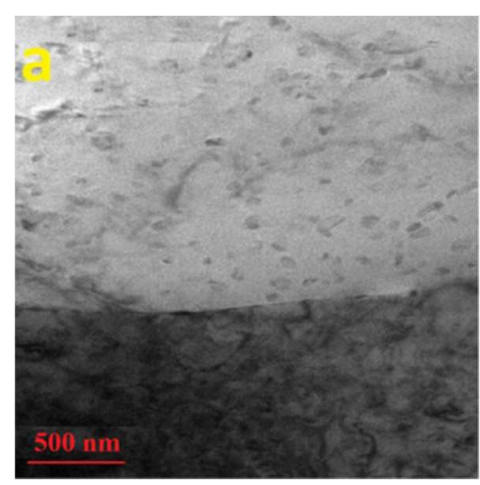

T6

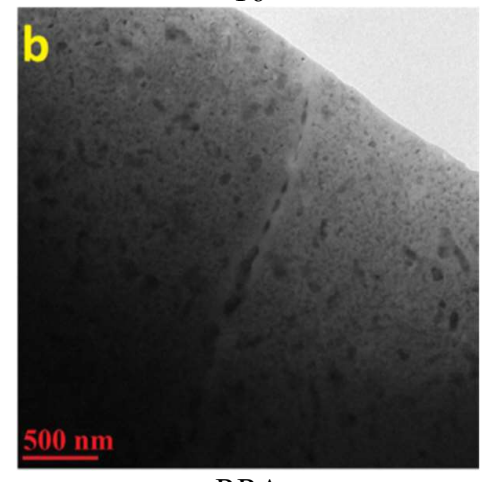

RRA

الثكل 8: صور المجر الالكتروني الماسح TEM لسبيكة 
[3] B. Cina.Patent US Patent 3856 584, 1974.

[4] F. Vianaa, A. M. P. Pinto, H. M. C. Santos and A. B. Lopes, "Retrogression and re-ageing of 7075 aluminium alloy:microstructural characterization," Journal of Materials Processing Technology, no. 92, pp. 54-59, 1999.

[5] George E. Totten and D. Scott MacKenzie, Handbook of Aluminum Physical Metallurgy and Processes, MARCEL DEKKER, INC, 2003.

[6] Gökhan Özer and Ahmet Karaaslan, "Effects of RRA heat treatment on the exfoliation corrosion (EXCO), intergranular corrosion (IGC), and electrical conductivity of AA7075 alloy," Materials and Corrosion, 2018.

[7] Guosheng Peng, Kanghua Chen, Songyi Chen and Huachan Fang, "Influence of repetitious-RRA treatment on the strength and SCC resistance of Al$\mathrm{Zn}-\mathrm{Mg}-\mathrm{Cu}$ alloy," Materials Science and Engineering A, no. 528, p. 4014, 2011.

[8] LI Guo-feng, ZHANG Xin-ming, LI Peng-hui and YOU Jiang-hai, "Effects of retrogression heating rate on microstructures and mechanical properties of aluminum alloy 7050," Transactions of Nonferrous Metals Society of China, no. 20, p. 935, 2010.

[9] Oskouei R. H. , Barati M. R. and Ibrahim R. N. , "Surface Characterizations of Fretting Fatigue Damage in Aluminum Alloy 7075-T6 Clamped Joints: The Beneficial role of Ni-P Coatings," Materials (Basel), 2016.

[10] Pastor A. and Svoboda H. G. , "Time-evolution of Heat Affected Zone (HAZ) of Friction Stir Welds of AA7075-T651," Materials Physics and Chemistry, 2013.

[11] TapanyUdomphol, Aluminum and its Alloy, Suranaree University of Technology, 2007.2

[12]W. F. Gale and T. C. Totemeier, Smithells Metals Reference Book, 8 ed., Butterworth-Heinemann, 2004.

[13]Z. H. Ji and Z. Z. Jing, "7075-T6 aluminium alloy exfoliation corrosion sensitivity and electrochemical impedance spectroscopy under stress," Materials Research Innovations, vol. 19, p. 230, 2015.
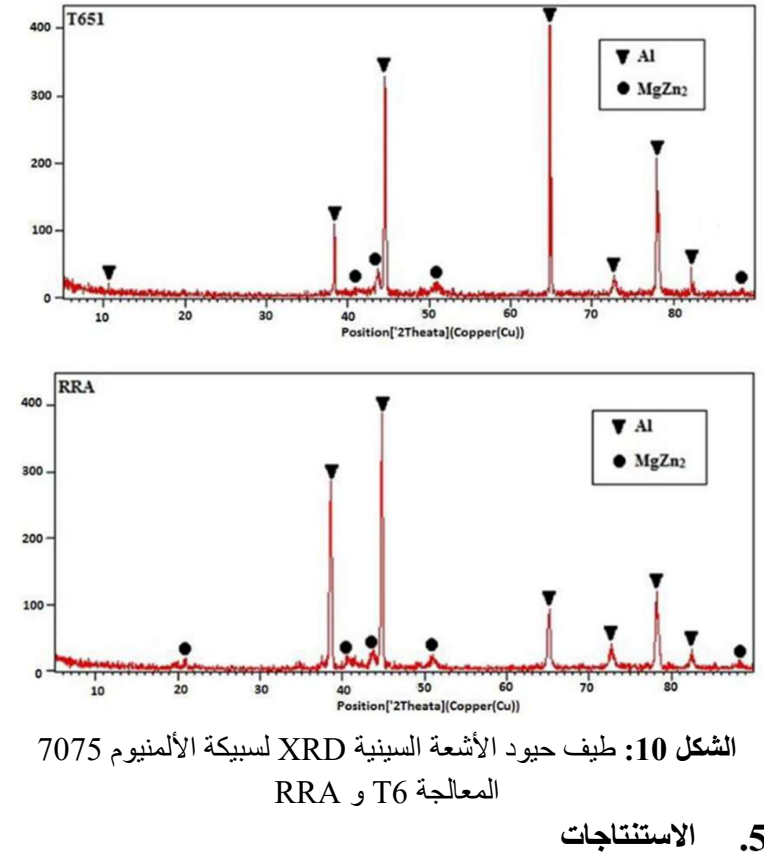

1

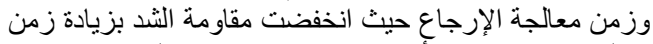

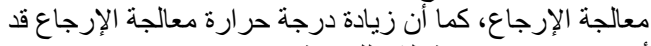
أدى لانخفاض مقاومة الثد للسبيكة.

2. وصلت سبيكة الألمنيوم 7075 لأعلى قيمة للمقاومة على الثد

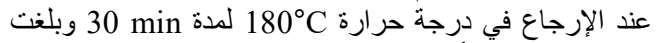

586 MPa

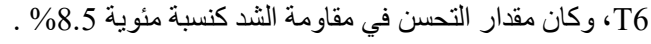

3. تغيرت صلادة برينل لسبيكة الألمنيوم 7075 بتغير درجة

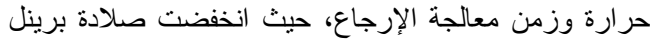

للسبيكة مع زيادة زمن معن معالجة الإرجاع الإعاع وكذلك مع زيادة درجة حرارة الإرجاع.

4. وصلت صلادة برينل لسبيكة الألمنيوم 7075 إلى أعلى قيمة لها

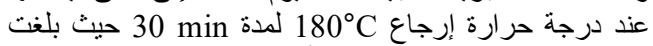

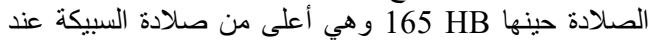

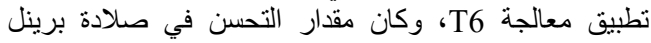

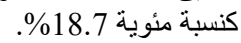

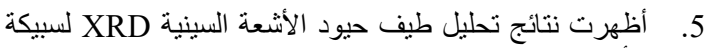

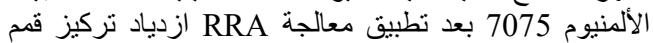

الطور MgZn

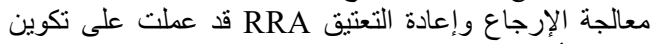

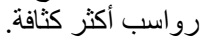

[1] A. Ghosh and M. Ghosh, "Microstructure and texture development of 7075 alloy during homogenisation," Philosophical Magazine, 2018.

[2] A. Mavropoulos and S. Skolianos, "Effect of Heat Treatments on the Corrosion Behavior of High Strength Aluminum Alloy," International Journal of Advanced Engineering and Management Research, 2018. 


\title{
Effect of Retrogression and Re-Aging Heat Treatment on Hardness, Tensile Strength and Microstructure of 7075 Aluminum Alloy
}

\author{
Mohamad Yehea Al nefawy ${ }^{*}$ and Fouad El dahiye ${ }^{2}$ \\ ${ }_{1}^{1}$ Department of Design and production engineering Al-Baath University, Homs, Syria, E-mail: yahya.nefawy@gmail.com \\ ${ }^{2}$ Department of Design and production engineering Al-Baath University, Homs, Syria, E-mail: FouadEldahiye@gmail.com \\ *Corresponding author: Mohamad Yehea Al nefawy and email: yahya.nefawy@gmail.com \\ Published online: 30 June 2021
}

Abstract- Retrogression and Re-Aging (RRA) Heat Treatment improves the tensile of aluminum alloys. In this research, to study the effect of Retrogression temperature and Retrogression time on the hardness, tensile strength and microstructure of 7075 aluminum alloy, have been applied. Retrogression treatments at different temperatures $180^{\circ} \mathrm{C}, 240^{\circ} \mathrm{C}$ and $370^{\circ} \mathrm{C}$ for $30 \mathrm{~min}$ and $90 \mathrm{~min}$. When the retrogression temperature was $180^{\circ} \mathrm{C}$ for $30 \mathrm{~min}$, the alloy has reached its highest hardness and tensile strength; they were respectively $165 \mathrm{HB}$ and $586 \mathrm{MPa}$. RRA treatment of aluminum alloy 7075 led to precipitate a smoother, more homogeneous, and denser phase compared to the T6 treatment.

Keywords- 7075 High Strength Aluminum Alloy, Retrogression and Re-Aging, Hardness, Tensile Strength, Microstructure, XRD 